\title{
ICA component selection based on sparse activelet reconstruction for fMRI analysis in refractory focal epilepsy
}

\author{
Borbala Hunyadi*§, Bogdan Mijovic*§, Simon Tousseyn ${ }^{\dagger}$, \\ Patrick Dupont $^{\dagger}$, Wim Van Paesschen ${ }^{\dagger}$, Sabine Van Huffel ${ }^{\star \S}$, Maarten De Vos ${ }^{*} \S$ \\ * SCD-SISTA, Department of Electrical Engineering, KU Leuven, Leuven, Belgium \\ ${ }^{\dagger}$ Department of Neurology, University Hospital Gasthuisberg, Leuven, Belgium \\ ${ }^{\ddagger}$ Neuropsychology Lab, Department of Psychology, University of Oldenburg, Oldenburg, Germany \\ $\S$ IBBT Future Health Department
}

\begin{abstract}
EEG-fMRI is a recently emerging tool that can be used in the presurgical evaluation of focal epilepsy patients. Standard analysis techniques rely on the principle that fMRI can provide accurate localization of hemodynamic changes corresponding to events observed on EEG. However, its applicability is limited as EEG does not always provide sufficient and reliable information on the timing of the epileptic activity. Therefore, there is an increasing demand for techniques capable of localizing the epileptic activity based solely on the fMRI time series. Independent component analysis (ICA) has been shown to separate epileptic activity in the fMRI from other neural sources, artifacts and noise. We propose here to automatically detect the epileptic component based on sparse reconstruction in the activelet basis. The algorithm was evaluated on a dataset of 10 patients. It is shown that the largest activation cluster of the identified component overlapped with the ictal onset zone (IOZ) in all 3 patients with sparse interictal spike timing. In the 7 other patients, the selected component either overlapped with the IOZ and/or the ictal hyperperfusion, or correlated with the EEG-derived time course of the interictal activity. We conclude that the proposed technique might be able to identify epileptic components without using EEG.
\end{abstract}

Keywords-ICA; activelet; fMRI; epilepsy;

\section{INTRODUCTION}

Functional magnetic resonance imaging (fMRI) combined with simultaneously recorded electroencephalogram (EEG) is an emerging technique for localizing epileptic activity as part of the presurgical evaluation. Standard analysis uses the timing of interictal discharges based on EEG to find regions with correlated blood oxygen level dependent (BOLD) signal changes recorded by fMRI within the standard general linear model (GLM) approach. However, the EEG analysis is time-consuming and it does not always provide reliable information on the epileptic events. Neural activity of deep structures is not visible on EEG, moreover, the severe artifacts due to the varying magnetic field of the scanner often make the interpretation of the EEG ambiguous. Finally, some patients might not show interictal activity during the limited time of the fMRI sessions. Therefore, there is a strong demand for techniques capable of localizing the epileptic activity without any information on its timing.
Rodionov et al. [1] used spatial ICA to extract spatially independent components (IC) from the fMRI. The ICs were automatically classified as BOLD related, artifactual or noise related and ICs corresponding to resting state networks (RSN) were ruled out visually. Epileptic ICs were identified among the remaining BOLD related ICs based on the EEG-fMRI activation maps and the EEG-derived temporal regressor. Successful identification of the epileptic activity showed the potential of ICA as a data-driven analysis tool. Nevertheless, the identification of the IC of interest still heavily relied on EEG.

Recently, activelets, a new wavelet dictionary matching BOLD characteristics was developed [2]. The activelets are constructed based on the linear approximation of the balloon model [3] of the hemodynamic response function (HRF). As the activelet waveforms follow the shape of the BOLD response of a transient neural activation, such transient activity will be sparsely represented in the activelet basis. The timing of transient, sparse neural activity can be estimated using a convex optimization algorithm. Lopes et al [4] applied activelets to detect transient activations related to epileptic spikes on each voxel time course, and used spatiotemporal clustering to gather voxels showing similar timing of activity. The cluster with the sparsest temporal activation pattern was appointed as epilepsy related. This method was shown to be very specific in detecting spikerelated fluctuations in the BOLD signal, and derived similar activation maps to the ones obtained by EEG-fMRI.

We present a method combining both techniques. We use activelets to sparsely represent the time courses of the different sources obtained with ICA. This allows to automatically select the IC containing transient activations and plausibly representing epileptic activity. The method is validated on a dataset of 10 patients with a well-defined IOZ after a full presurgical evaluation including ictal single photon emission computed tomography (SPECT). The results are compared to the subtraction ictal SPECT coregistered to MRI (SISCOM) hyperperfusion maps, and to the localization of the IOZ. 


\section{Methods}

\section{A. Data acquisition and preprocessing}

Functional images were acquired in 10 patients with refractory focal epilepsy using a $3 \mathrm{~T}$ MR scanner with a whole brain single-shot $\mathrm{T} 2 *$ gradient-echo Echo Planar Imaging sequence $(\mathrm{TE}=33 \mathrm{~ms}, \mathrm{TR}=2.5 \mathrm{~s} / 2.25 \mathrm{~s} / 2.2 \mathrm{~s}$, voxel size 2.6/3/2.6 mm). The images were realigned, slice-time corrected, normalized to MNI space and spatially smoothed with an isotropic Gaussian kernel of $6 \mathrm{~mm}$ FWHM using SPM8 software (Wellcome Department of Cognitive Neurology, London, UK). Scalp EEG was simultaneously recorded using a 24,32 or 36 channel MR compatible electrode set. The patients were resting with closed eyes during the recording sessions, which lasted on average 12 minutes, ranging from 10 to 22 minutes. The patients were selected based solely on the criterion that clinically concordant interictal epileptic spikes were recorded by the EEG inside the scanner. The timing of interictal epileptic spikes was visually marked by a neurologist. Ictal SPECT scans were also performed on these patients, as part of the routine presurgical evaluation procedure. The IOZ was determined by a neurologist as the overlap between the SISCOM hyperperfusion maps $(z>1.5)$ results and the effective or intended resection zone.

\section{B. Sparse representation of BOLD signal in activelet basis}

The neural activity of interest consists of $k$ interictal epileptic spikes with amplitudes $A_{k}$ and onsets $t_{k}$. The fMRI measures the BOLD signal changes as a result of this neural activity. The hemodynamic system linking the neural activity to the BOLD signal is denoted by $h$, and is commonly assumed to be linear and shift-invariant. The BOLD signal recorded by the fMRI can be written as follows:

$$
y(t)=\sum_{k} A_{k} h\left(t-t_{k}\right)+\epsilon(t),
$$

where $\epsilon(t)$ is a unknown noise term comprising noise, baseline, drifts, physiological artifacts and possibly other unrelated neural activity. Our goal is to recover the activity of interest from the noisy fMRI data.

Activelets are a recently developed dictionary of wavelet basis functions [2]. The activelets are constructed based on the linear approximation of the balloon model of the HRF. Given that the neural events are sparse in time, the linearity assumption holds and the transient BOLD signals can be sparsely represented in the activelet basis. Let $\phi$ be the overcomplete dictionary matrix containing the basis functions of the undecimated activelet transform. Then the estimated neural activity $x$ will be given by $x=\phi \beta_{0}$, where $\beta_{0}$ is the solution of the convex $l_{1}$ optimization problem:

$$
\min _{\beta}\left(\frac{1}{2}\|y-\phi \beta\|_{2}^{2}+\lambda\|\beta\|_{1}\right)
$$

The regularization parameter $\lambda$ controls the trade-off between the sparsity of the solution and the reconstruction error, a higher value favoring a sparser solution. $\phi$ is a size $T \times P$ matrix, where $\mathrm{T}$ is the length of the time series; $\mathrm{P}$ is the number of decomposition scales and was set to 3 in this work as in [4]. The minimization problem in (2) was solved by the Homotopy algorithm [5].

\section{Independent component analysis}

The measured fMRI time series is the result of the mixture of several different ongoing neural activity, artifacts and noise. Assuming that the activity of interest has a fixed spatial pattern which is independent of the ones related to other underlying processes, ICA separates them in the following way:

$$
U=V Z
$$

where $U \in \mathbb{R}^{n \times m}$ is formed by the component voxel values where $n$ is the number of independent spatial components and $m$ is the number of voxels; $Z \in \mathbb{R}^{n \times m}$ contains the measured fMRI time course of all voxels and $V \in \mathbb{R}^{n \times n}$ is the unmixing matrix. The columns of the mixing matrix $M=V^{-1}$ are the time courses associated with each IC.

The infomax algorithm was used to estimate the ICs. The temporal dimension was reduced by principal component analysis in order to obtain fewer number of ICs. The optimal number of ICs was chosen automatically for each patient using the MDL criteria, resulting in $52.5 \pm 13.6$ components.

\section{Proposed method}

Our goal is to recover the signal related to epileptic activity from the noisy fMRI data. The interictal epileptic activity is assumed to comprise transient activations with sparse timing. We apply spatial ICA on the fMRI data to extract epilepsy related activity. ICA performed on fMRI data generally results in a high number of ICs, and the selection of the IC of interest is challenging. We propose to determine which IC corresponds to the epileptic activity by means of the activelet framework. It is assumed that the epilepsy related IC contains spikes, and no other IC does. In other words, its time course contains a segment (i.e. the BOLD signal corresponding to an epileptic spike) which matches best the hemodynamic response encoded in the activelet dictionary. The epileptic IC hence will have a sparser representation in the activelet basis than any other IC. The IC of interest is thus obtained in the following manner. The regularization parameter is set high and the reconstruction algorithm in (2) is run on each IC time course. When representation at the sparsity level determined by $\lambda$ is not possible, no spikes are detected, i.e. all activelet coefficients are zero. If this is the case for each component, $\lambda$ is lowered and the algorithm is run again. The first time when the algorithm detects an event for an IC time course, 

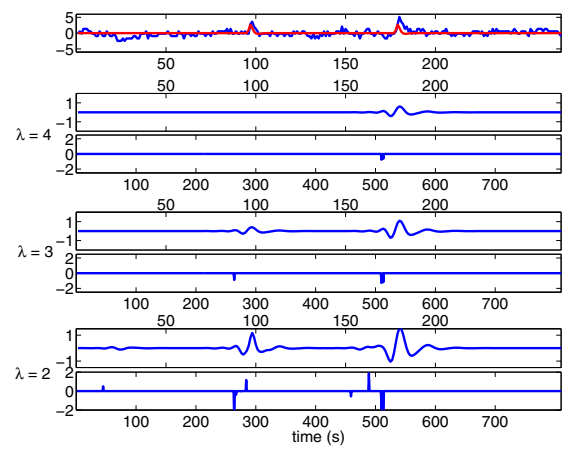

Figure 1. The top panel shows the time course of the most significantly activated voxel according to the GLM analysis (blue line) and the timing of the interictal epileptic activity convolved with the HRF (red line). The three figure pairs show the reconstructed signal (upper figures) and the activelet coefficients (lower figures) obtained for different $\lambda$ parameters.

the IC with the sparsest possible activelet reconstruction is found.

Fig. 1 illustrates how the regularization parameter $\lambda$ controls the trade-off between the sparsity and the reconstruction error of the solution. The time course of the epileptic IC obtained for the $2^{\text {nd }}$ run of patient 6 was reconstructed in the activelet basis for different $\lambda$ values. Note that for a high $\lambda$ only one of the spikes are reconstructed, while for a low $\lambda$ signal fluctuations unrelated to epileptic activity are also modeled. Setting $\lambda$ high avoids the the reconstruction of such false positives events. Moreover, preprocessing the raw voxel time courses in order to reduce signal fluctuation will facilitate better and sparser activelet reconstruction. Fig. 2 shows the reconstruction error of the ideal spike time course as the function of the sparsity of the activelet coefficients obtained for the time course of the maximally activated voxel according to the GLM analysis and the one obtained for the IC corresponding to the epileptic activity. The arrows are indicating the points on each graph with the highest sparsity of reconstruction, with which both spikes are already retrieved. Note that this is achieved with a sparser solution for the IC time course than for the voxel time course, additionally confirming the validity of ICA.

\section{E. Validation criteria}

The obtained component voxel values were converted to $\mathrm{z}$-scores by extracting the mean and dividing by the standard deviation. A threshold of $z>2$ was used to create component activation maps. The results were validated in two steps. First, it was determined, whether ICA is capable of blindly extracting the epileptic activity. Adapting the criteria used in [1], interictal spike related ICs were identified based on (1) significant $(p<0.05)$ correlation between the EEG spike time course convolved with HRF and the component time course, and (2) overlap between the IOZ and the largest activation cluster of the component. These ICs are likely to be related to epilepsy. Note however, that

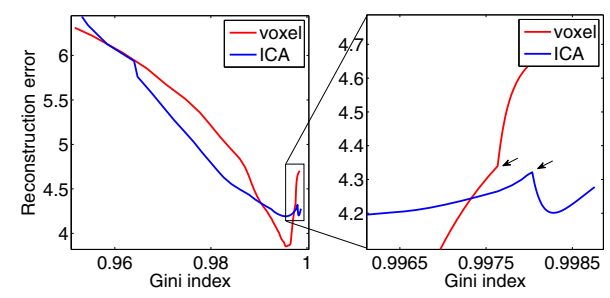

Figure 2. Reconstruction error as a function of the sparsity of the representation, measured by the Gini index ( [6], a higher value indicates a sparser signal). The curves were obtained by varying $\lambda$. The reconstruction error is computed as the mean square error of the reconstructed signal compared to the true spike time course. The arrows mark the points with the highest sparsity of reconstruction, with which both spikes are already retrieved. Note that this is achieved with a sparser solution for the IC time course than for the voxel time course.

EEG information might be unreliable, thus, ICs showing no temporal correlation with the spike timing could also be related to epileptic activity. In the second step, the goodness of the IC selected based on sparsity in the activelet basis should be assessed. This was done by comparing the largest activation cluster of the selected ICs to the SISCOM hyperperfusion maps and the IOZ for each patient.

\section{RESUlTS}

We defined 2 patient groups according to the sparsity of their spike time course, by determining the minimum interval between 2 consecutive spikes per session. A liberal threshold of $5 \mathrm{~s}$ was used to include a patient into group 1 . As a result, 3 patients were included in group 1 , and 7 patients with less sparse time courses formed group 2.

ICA was capable of extracting spike-related components showing both temporal and spatial concordance with the criteria described above in 9 on 10 patients. There were ICs showing either temporal or spatial match in the remaining 1 patient belonging to group 2 .

The largest activation cluster of the IC selected by the activelet based algorithm overlapped both with the SISCOM hyperperfusion map and the IOZ in all 3 patients in group 1. In 2 out of 3 patients, the selected IC had even the largest overlap with the IOZ among all components, and its time course also showed temporal correlation with the spike timing. Fig. 3 shows the IOZ and the activation maps derived by our method for one of the patients in group 1 . In group 2 , the activation map of the selected IC overlapped with the SISCOM hyperperfusion map in 5 on the 7 patients; and overlapped with the IOZ in 2 on 7 patients. The selected IC was further analyzed for those 2 patients, where no overlap with SPECT was found. The time course of these ICs correlated with the timing of the interictal spikes convolved with the HRF function $(p<0.01$ and $p<0.001)$. Thus, it seems that despite of showing no spatial overlap with the gold standard, these ICs might be related to epilepsy as well, possibly representing fast propagated activity. 


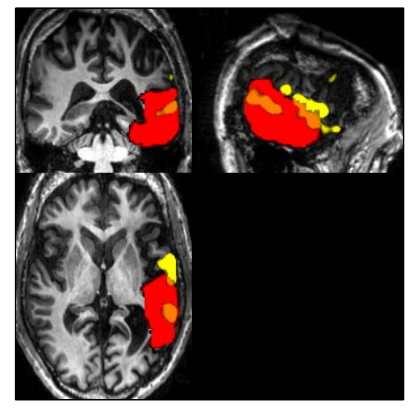

Figure 3. Overlap (orange) between activation maps derived by the proposed method (yellow) and the IOZ (red) for a patient in group 1.

\section{Discussion}

Several studies have shown the clinical usefulness of EEG-fMRI in localizing interictal epileptic activity in the brain. The main disadvantage of this technique is that it relies on the timing of epileptic activity as read on the EEG, which is not always available or reliable. We proposed a method capable of inferring to the localization of the epileptic activity without any information on its timing.

Our method works in two stages. First, ICA is applied to separate different neural and non-neural sources from each other. We assume that at least one of the ICs is related to interictal epileptic activity. Previous studies confirm the validity of such an assumption [1]. In the second stage the IC related to interictal activity is selected. The IC time courses are analyzed, and the one with the sparsest possible representation in the activelet basis is chosen. Note that sparser reconstruction can be expected for the IC time course compared to the raw voxel time course due to the higher signal to noise ratio.

The second stage of the algorithm relies on the assumption that the timing of interictal activity is sparse. The patients were divided in two groups according to whether their spike time course met this criterion or not. The epileptic IC selected by our method was in accordance both with SPECT and the IOZ in all patients in group 1. Moreover, there was either spatial or temporal match with SPECT and the timing of interictal spikes, respectively, in all patients in group 2. Therefore, the proposed technique might be able to provide useful insight into the epileptic activity even under mild conditions.

The results suggest that identifying sparse ICs can outperform the identification of sparse voxel time courses, as reflected by $93 \%$ and $69 \%$ spatial match in the sparse and non-sparse groups respectively, reached in [4]. However, it is hard to compare methods on different datasets and our current sample size is rather small.

These preliminary results nevertheless illustrate two potential advantages of our method. First, it is entirely auto- matic: the number of ICs and the parameter $\lambda$ are both datadriven as opposed to [4], where both the number of clusters and $\lambda$ were arbitrarily chosen. Second, it is computationally efficient, as the sparse reconstruction algorithm in Eq. 2 only has to be performed on the IC time courses instead of on all voxel time courses, which is orders of magnitude larger.

The ICA stage of the proposed method could be further improved. We restricted ourselves to the identification of only one sparse epilepsy related IC, however, several spike related ICs were found in case of certain patients. It should be investigated, whether these ICs are truly independent (corresponding to different propagated activity) or they are erroneously separated. There are robust techniques available to analyze the stability of ICs, which could help to optimize the number of ICs. Furthermore, several ICs corresponding to RSNs were found. These ICs could be automatically rejected a priori, along with ICs corresponding to different sources of artifacts and noise [1]. Finally, future research will aim at converting the current two-stage algorithm into a single constrained ICA algorithm, where the estimated ICs are enforced to be sparse in the activelet basis.

\section{ACKNOWLEDGMENT}

Research supported by: GOA MaNet, $\mathrm{CoE}$ EF/05/006 (OPTEC), PFV/10/002 (OPTEC); FWO: G.0341.07 (Data fusion), G.0427.10N (Integrated EEG-fMRI), G.0108.11 (Compressed Sensing); IWT: TBM070706-IOTA3, TBM080658-MRI (EEG-fMRI), IBBT; IUAP P6/04 (DYSCO, 2007-2011); Neuromath (COST-BM0601); Alexander von Humboldt stipend

\section{REFERENCES}

[1] R. Rodionov, F. De Martino, H. Laufs, D. Carmichael, E. Formisano, M. Walker, J. S. Duncan, and L. Lemieux, "Independent component analysis of interictal fmri in focal epilepsy: Comparison with general linear model-based eegcorrelated fmri," NeuroImage, vol. 38, no. 3, pp. 488 - 500, 2007.

[2] I. Khalidov, J. Fadili, F. Lazeyras, D. V. D. Ville, and M. Unser, "Activelets: Wavelets for sparse representation of hemodynamic responses," Signal Processing, vol. 91, no. 12, pp. $2810-2821,2011$,

[3] R. B. Buxton, E. C. Wong, and L. R. Frank, "Dynamics of blood flow and oxygenation changes during brain activation: The balloon model," Magnetic Resonance in Medicine, vol. 39, no. 6, pp. 855-864, 1998.

[4] R. Lopes, J. Lina, F. Fahoum, and J. Gotman, "Detection of epileptic activity in fmri without recording the eeg," NeuroImage, vol. 60, no. 3, pp. 1867 - 1879, 2012.

[5] D. Donoho and Y. Tsaig, "Fast solution of $\ell_{1}$-norm minimization problems when the solution may be sparse," Information Theory, IEEE Transactions on, vol. 54, no. 11, pp. $4789-4812$, nov. 2008 .

[6] N. Hurley and S. Rickard, "Comparing measures of sparsity," in Machine Learning for Signal Processing, 2008. MLSP 2008. IEEE Workshop on, oct. 2008, pp. $55-60$. 